RECENES BY TIC FE $1299 \%$

Pregented at the IETE Nucloax

iscience Sympos lum, oMiami, FL

Décember $5-8,1972$
(BAL DOCUMENT TH 360)

$\operatorname{CoN}+\% 21282--14$

(4)

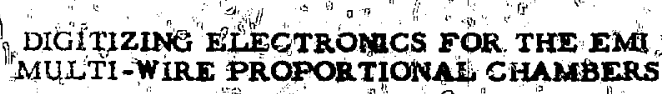

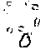

of

?

sit.

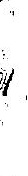




\title{
DIGITIZING ELECTRONICS FOR THE EMI MULTI-WIRE PROPORTIONAL CHAMBERS ${ }^{\star}$
}

\author{
E. BInnall, F. KIrsten, K. Lee, C. Munnally \\ Lawrence Berkeley Laboratory \\ liniversity of California \\ Berkeley, Californta
}

\section{Sunmary}

A complete system of electronics for digitizing pulses from a larce array of multi-wire proportional counters is described. The system is based on the method of coupling the chamber pulses onto delay 1 ines developed by Perez-Mendez. All three wire planes of each chamber are digitized, giving positiona) information for each avalanche in the chamber on three coordinates, $x, y$ and a redundant coordinate.

The systen will be used with an array of 30 chambers each one meter square, which wil1 serve as an External Mon Identifier (EMI) for the 15-foot diameter bubble chamber in the neutrino beam ine at HAL. Up to 16 events can be digitized in a 60 ps beam spill.

\section{Introduction}

A 15-foot diameter bubble chamber is being built for the neutrino beam line at the National Accelerator Center. Under a plan proposed by Stevensun and Peterson, 1 the bubble chamber w111 be augmented by a layer of Multi-wire, roportional Chambers (MAPC) surrounding a portion of the bubbie chamber periphery. The purpose of this layer is to penit tdentification, external to the bubble chamber, of muons involved in bubble chamber events. The system of MAPCS is therefore referred to as an External thon Identifier (EMI).

Thic Initial phase of this plan will have 30 square meters of MHPC, consisting of 30 individual chambers each one meter on a side. ${ }^{2}$ Each chamber will have 3 coordinate axes digitized--X, $Y$ and $U$. $U$ is a redundant plane whose coordinate axis is rotated $45^{\circ}$ with respect to $\mathrm{K}$. The location of a charged particle passing through a chamber will be digitized to a resolution of t5 $\mathrm{mm}$ on each coordinate axis. During a 60 us beam 5 p Th1, an average of 5 events is expected in each chabber.

The MAPC readout is based on the electromagnetic delay 11 nes developed by Perez-Hendez. ${ }^{3}$ For this application, delay i ine digitization has some attractIve features compared to other dfgftizing means, such as the logic channel-per-wire. ${ }^{4}$ first, it tends to reduce the cost of electronics, since only seven channels of electronics are necessary to dfaitfze all three coordinates of a chamber. Each channel is reasonably inexpensive, in this case partly because of the relaxed resolution requirements. Amplifierper-wire logic usually is attached only to one plane of a chamber. In contrast, delay IInes can be coupled to all inree planes, hence the number of chambers required and the chamber cost is reduced.

\footnotetext{
This work was done under the auspices of the U.S.
}

Atomic Energy Cormission.

\section{Relationship of Elecirontes to the Chambers}

Ftgure 1 shows a schematic view of a chamber and Its three delay itnes, one each for $x, \gamma$ and $U$ cocrdinates. The wires of the three planes are extended a suffictent distance to establish a capacitive coupling of each wire onto the delay line. An event (avalanche) on a particular wire results in a voltage puise Deing introduced onto the delay line at that point. The pulse then propagates to the ends of the delay line at a velocity of the order of $0.2 \mathrm{~mm} / \mathrm{ns}$. Seven digitizer channels are used with each MWPC: one on each end of the three delay lines, plus one to record the "prompt" (undelayed) times of the chamber events. Each digitizer channel records the time at which it recelves each of up to 16 pulses during a beam burst. Since there are 30 MAPCs, a tota? of 210 digitizer channels is used. At the end of a beam burst, they deliver all the data they have collected (up to $16 \times 210=3360$ words) during the burst.

A sultably designed computer program can analyze the collected dita from each chamber to determine the location and time of occurrence of each event within that chamber. The Appendix gives some detal1s of the analysis procedure.

\section{Components of the Digitizer Chatn}

The pulses at the delay-7ine outputs generally have amplitudes of a few millivo?ts and rise-times of the order of $100 \mathrm{~ns}$. The delay ilnes have characterIstic Impedances of about 1000 ohms. The preampl ffier is mounted phystcally adjacent to the delay line, and amplifies the signals before they are transmitted from the chambers to the discriminators on 50-ohm coaxial cable. The discriminators deveiop timing pulses whose leading edges are an accurate measure of the time-ofarrival of the deiay line pulse. The timing pulses are delivered to the digitizers. Thay develop and store binary numbers whose magnitudes are related to the times-of-arrival.

\section{Preamplifters}

The spatial resolution of the digitizing channels depends on how well the times-of-arrival of the pulses can be measured. This in turn is strongly dependent on the signalinoise ratio at the timing discriminator. The relationship is approximately:

$$
T_{j}=\frac{E_{n}}{5} \text {, }
$$

where $T_{j}=$ tfming error ( $j+t$ ter) introduced by the presence of the noise, seconds;

$E_{n}$ - notse superimposed on the signal,
volts; and

$S$ - the slope of the sfgnal waveform, volts/sec. 
Both $E_{n}$ and $T_{j}$ are expressed in the same way-e.g., rmls, peak or FWHM. For many pulses, $S$ can be expressed as $S=1.25 \varepsilon / \alpha$, where $E$ is the peak amplitude, and $\tau$ is the 10-90\% rise-time.

The signal/nolise ratio is determined mainly by the characteristics of the input stage of the preamplifier.

Al though the spatial requirements for this expertment are relaxed, some experiments require resolution of a fraction of a millimeter. These preamplifiers have therefore been designed for low input noise to accomodate a wide range of applications. An amplifier bandwidth of $3 \mathrm{MHz}$ is sufficient for the signals typical of these delay lines.

A terminated deiay ?ine represents a source with impedance $20 / 2$, where $20 / 2$ is typically 500 ohms. The resistive noise from a 500 obm resistor at room temperature in a $3 \mathrm{HHz}$ bandwidth system is $5 \mathrm{\mu V}$ rms. The equivalent input notse from preamps using lownoise bipalar transistors has been measured to be $8.5 \mu \mathrm{V}$, when connected to terminated 1000 -0hm delay 1ines. Since notse voltages add vectorially the noise contribution of the amplifier itself is calculated to be 7 py.

Low-noise field-effect transistors (FETS) are jenerally less suitable than bipolar transistors in this applifcation, because of the low impedance of the source. For example, a preampliffer using low noise FETs was found to have a noise performance a factor of two poorer than the one using bipolars.

Some low-cost linear integrated circuits are now available. A few of these have adequate gain-bandwidth product and reasonably low notses. The circuit of a preamplifiter using a Fairchild type 733 is shown in Fig. 2. It exhibits an equivalent input noise of $13 \mu \mathrm{V}$ when connected to a delay line. While this is not as good as the discrete bipolar design, it is adequate for many purposes and results in a smaller printed circult board size. This is the design used in the ENI project.

\section{D1scriminator}

Figure 3 shows a functional block diagram of a discriminator channe1. Input sfgnals from the preamplifier are differentlated to form a zero-crossing pulse, and then amplified by a factor of 10. The times at which the pulses cross the base-ilne are detected by the zero-cross detector, which triggers the fast monostabie. The final stage then delivers a TTL-compatible output pulse. Seven identica? channe7s are housed in a NIM module.

The zero-crossing detector uses an RCA CA3086 integrated circuit in combination with an 1 N3717 tunnel diode (TD) to sense the zero-crossing. Each integrated circuit has one differential pair and three independent transistors. Since all are contained on the same monolithi: chip, the transfstors maintain their matched characteristics over a wide temperature range. The circuit is therefore considerably stabler than if discrete transistors were used. The only adjustment provided is a tunnel-diode bias control.

A simpliffed circuit of the zero-cross detector is in Fig. 4a, and the tunnel diode characteristic and load line in Fig. $4 b$, With no input signal, the current supplied by current source Q3 is adjusted until the To is operating at point of $A$ of Flg. 40 . Note that point $A$ lies on a load line that passes through point $D$. A positive-going input to $Q_{1}$ diverts current from $Q_{2}$, thereby moving the To operating point toward noint $B$. If the input becomes more positive than the threshold level, the TD switches from B to C. As the input signal returns toward zero, current $Q_{1}$ decreases, and the TD operating point moves toward $D$. If the bias was initially set as described above, the TD switches from $D$ to $A$ just as the input crosses through zero. The switching of the TD from D to $A$ triggers the monostable and generates the output pulse.

The transistor $Q_{5}$, between $Q_{2}$ and the $T D$, has the important function of reducing the internal feedback due to the Miller effect. This afds in deriving a precise timing point from a relatively slow-moving input signal, and has been shown to reduce the timing jitter. The timing jitter measured wfth an input having $100 \mathrm{~ns}$ rise-tfme is less than $1 \mathrm{~ns}$. The time-slewing is within $\pm 2 n s$ for an input amplitude range of 50 to $1500 \mathrm{mv}(30: 1)$.

\section{Digitizer Module}

The digitizer is a CAMAC module that can digftize the times-of-arrival of up to 16 pulses on each of seven inputs. The tine-of-arrival of each pulse is digitized by storing "on-the-fly" the contents of a scaler that is counting externally supplied clock pulses. The digitfzer can operate with clock frequencles up to $28 \mathrm{MHz}$, hence can measure the time-ofarrival to $\pm 36 \mathrm{~ns}$.

The block diagram of Fig. 5 shows the organization of the digitizer. Clock pulses are counted by a 12-bit synchronous scaler. The contents of the scaler are available to each of the seven independent channels. As each signal pulse enters a signal input, it causes the contents of the scaler $t a$ be transferred to the corresponiing Temporary St. $r e$. This transfer may be done only within a certain acceptance interval during each clock period, which, at a clock frequency of $28 \mathrm{MHz}$, is approximately 20 ns: The input synchron1zer insures that this transfer is triggered only at an appropriate phase of the clock period.

The transfer of the data from temporary store to scratchpad memory may take up to $100 \mathrm{~ns}$, since input pulses are not expected to be closer together than that. This permits the use of scratchpads that have longer minimum acceptance times than the temporary stare. The scratchpad memories are each composed of three 16x4 bit read/write integratedcircuft memories, with address decoding (IC Type Am31L01). The memories are organized on a lastin-first-out basis. A 4-bit UP/DOWN counter with MAX/MIN carry-out (SN74191) is used as an address scaler for each scratchpad. Durtng digitizing, it is incremented each time a word is stored. If 16 words are stored, the MAX/MIN carry is used to disable the signa1 input. During readout, the address scaler is decremented, and the MAX/MIN carry signals when al1 "fresh" words have been read out.

Readout Process. It is generally the case that a random number of pulse inputs are received during the interval that the moduse is actively digitizing. Thus a random number of words will be stored in each scratchpad memory during this interval. The digftizer 
is designed to read out oniy the words that have been collected since the last previous readout. The module uses the Q-controlled Stop mode* of block transfer to move the data, via the CAMAC Dataway, to the computer or storage device. The Stop mode is especially useful for transfers where the module controls the number of words to be transferred. The digitizer module behaves as though the Read command accesses a single register through which all words are transferred. This register is the only one in the module using $F(0)$, hence subaddresses can be ignored for $F(0)$. A controller can access arrays of digitizers by using the algorithm:

\section{Step 1. Set up Dataway command $N(1) \cdot F(0) \cdot A(0)$ where $N(i)$ is the station containing the first module in the array.}

Step 2. Execute a Dataky cycle (module ignores subaddress).

Step 3. Test Q received during this Dataway cycle.

a) If Q=1: Transfer data to storage; increment subaddress (optional); repeat Step 2 .

b) If $Q=0$, chis module is finished, so go to the next: Do not transfer data; increment station number, N, reset subaddress to zero (optiona1): repeat Step 2 .

Some CAMAC Branch Drivers** are equipped to execute this algorithm in hardware. The optional subaddress manipulation makes this algorithm compatible with many rodules designed for Address Scan mode.

During the transfer of data from a module, thr. Read Priorisy Sorter (see FIg. 5) controls the order In which digitizing channels are read out. It first enables the transfer of a single module-identification word (priority 7 ). During this transfer, the module gives a Q-response of ${ }^{\prime} O{ }^{\prime}$ if there are no data words stored in the scratchpads. If there are stored words, the Indtidual channels are read out in order, each channel contributing only the number of data words gathered since the last readout. Since the memory address scalers are decremented during this process, each finally resting at memory address 0 , a completely read out module is ready for the next digitizing interval.

Data Identification. Generally, several modules will be read into a single block of computer memory. Since each contributes a variable number of words, some means of Identifying the origin of each word must be provided. Fortunately, the scaler is only required to be 12 bits. Therefore, 4 bits are

\footnotetext{
Fsee the CAMAC Specification TID-25B75' (EUR-4100e), Sec. 5.4.3.3. In the Stop mode the nodule generates a $Q=1$ response to a Read command [typical1y $F(0)$ ] as long as it has a meaningfud data word to transfer on that command. It generates a $0=0$ response after all its words have been transferred.
}

* For example, the EG\&G BD-0115 or the Los Alamos micro-programed Branch Driver. ${ }^{6}$ available for identification within the 16-bit word size that is convenient for many computers. One bit is used to unlquely identify the module Identification word shown in Fig. 6a. This word, the first word read from each module, carries an B-bit module identification code. A1T other words use the format shown In FIg, 6b. Three bits in each data word show the channel number from which 1 t originated. Thus, in the block of memory, the sets of words contributed by individual modules are delimited by words carrying i's in the most significant bit position. These identify the module contributing the words in the inmediately following set. Within this set, Identification of the channel originating each word is carried in the word itself.

\section{Clock Module}

The CAMAC clock module provides a common time base for up to 100 digitizers, and helps to synchronize the variaus phases of the data gathering and readout. Its relationship to the other components is shown in Fig. 7. Its two output signals, Clock and Write Pemit are bussed to all the digitizer modules. The state of Write Permit determines whether the digitizers are in the digitizing (writing-into-momory) state. The clock output is a train of $28 \mathrm{MHz}$ pulses derived from a crystal oscillator. These pulses are connected by a scaler in each digitizer which can then measure the times of-arrival of signal pulses in terms of the number of clock pulses received up to that tine.

Arrival of a Start of Ream Spill Sync pulse from the accelerator causes the Write Penmit signal to become true, and triggers the start of the clock train. The number of clock pulses in the train issued is preset by CAMAC comand or by front panel controls. At the end of the train, Write Permit goes False, and the Clock module ralses its ' $L$ ' flag to notify the computer of the end of the digitizing period.

\section{Fiducial Module}

Each delay Jine can be fitted with two extra input ports, one near each end of the line. These are used to infect simulated chamber pulses onto the delay ilne. The simulated pulses can be digitized and the results checked to make sure all components are workIng properly.

The CAMAC fiducial module generates fisticlal triggers wider CAMAC control. These are sent to all the chambers to cause the stmulated puises to be generated.

\section{Complete System}

A block diagram of a portion of a system is shown in FIg. 7. Figure 8 shows the sequence of major states of the system in processing data from a bean sp11]. The clock module is erimiled, via CAMAC, to accept a Beam Spill Sync pulse. The arrival of the Sync pulse starts the digitizing phase. The computer is notified of the end of this phase by a Look-at-Me from the clock module. The computer then initlates the algorithm described above to transfer the data collected by the Jigitizers into the computer menory.

Several system testing features are included by which varlous parts of the system can be checked. For example, with the clock disabled, the scalers in the Individual digitizer modulas can be loaded with a predetermined number by CAMAC command. Then the scratchpad memortes in chosen channels of each otgttizer can be caused to store this number. The numbers 
so stored can be read out and checked against the predetermined number. Further back in the systens. the discriminators can be triggered to produce output pulses on all channels simultaneously. These are digitized under dynanic conditions-i.e., clock running. All digitizers should have stored the same number to within the usual scaling error of plus or minus one count. If the fiducial module is triggered to generate simulated chambsr pulses, then the discriminators are also included in the test.

\section{Acknowledgments}

We are happy to have been able to work with a group of Lawrence Berkeley Laboratory-University of Hawait experimenters while developing this system. This group includes L. Stevenson, V. Peterson, S. Parker, F. Harris, V. Stenger and J, Crosetti.

\section{Appendix}

Figure I shows that each MHPC chamber will develop a pulsti on each of seven channels for every event. Figure 9 illustrates the significance of the pulses. Three time diagrams are shown, one for each delay line. Time increases from top to bottom. The abscissae represent distances along the delay lines. An event that occurs at location $x_{1}, Y_{1}, U_{1}$ and at time $t=t_{C l}$ is depicted. The line segments at angle a with the horizontal represent the trajectories of the pulses on the delay ifnes. As a result of this event, pulses arrive at the ends of the delay lines at times $t_{A 7}, t_{B T}, t_{D 7}, t_{E I}, t_{F]}$, and $t_{G]}$. The time $t_{A 1}$ is recoroded in digitizer channel $A, t_{B 1}$ in $B$, $t_{C 1}$ in $C_{\text {. }}$, etc. The location of the event can be determined by appropriate mathematical operations on any two of $t_{A T}$, $t_{B 1}$, and $t_{C l}$ ( $X$ coordinate); and any two of $t_{\mathrm{Cl}}, t_{01}$, and $t_{F 1}$ (Y coordinate).

For example, $X_{1}$ can be calculated by any of the following:

$$
\begin{aligned}
x_{1} & =\left(t_{A 1}-t_{C 1}\right) v, \\
& =1 \cdot x-\left(t_{B 1}-t_{C 1}\right) v, \text { or } \\
& =\frac{L_{X}}{2}-\frac{t_{B 1}-t_{A 1}}{2} v,
\end{aligned}
$$

where $v=$ velocity of propagation of pulses in the delay line, and

$L_{x}=$ physical length of $x$-plane delay IIne.

The reconstruction using equation ( 3 ) is advantageous for the following reason: a shift in the measured time difference $\left(t_{B 1}-t_{A 1}\right)$ of $\Delta t$ represents a shift in coordinate of the event of $\Delta X=\Delta t / 2 v$. If (1) or (2) are used, it represents only $\Delta x=\Delta t / y$. Thus a given time resolution in the measuring device results twice as good a spatial resolution if pulses from the two ends of the line are digitized (equation 3), rather than pulses from one end and the prompt puise (equations 1 and 2 ).

The pulses $t_{F I}$ and $t_{G I}$ are used to determine the $u$ coordinate. It can be $\mathrm{GSec}$ to resolve ambiguities that arise if two events occur simultaneously in the chamber.

Figure 10 shows a case in which two events occur at time $t_{C_{1}}$, one at $x_{1}$ and one at $x_{2}$. Note that channels $A$ and $B$ each recelve two pulses, and that the pulses at $t_{A 1}$ and $t_{B 1}$ (subscripts assigned in timeof-arrival sequence) are not associated with the same event. Given the times-of-arrival $t_{C_{1}}, t_{A 1}$ " $t_{A 2}, t_{B 1}$, and $t_{B} 2$, a reconstruction of the event in the $x$-coordinate can be made by noting that $\left(t_{B 2}-t_{C 1}\right)+\left(t_{A 1}-t_{C i}\right)=T$ and that $\left(t_{B 1}-t_{C 1}\right)+$ $\left(t_{A 2}-t_{C l}\right)=T$, where $T=\frac{L x}{V}$ is the total propagation delay of the line. Therefore the coordinates can be found:

$$
\begin{aligned}
& x_{1}=\frac{L x}{2}-\frac{t_{B 2}-t_{A 1}}{2} \text { and } \\
& x_{2}=\frac{L x}{2}-\frac{t_{B 1}-t_{A 2}}{2} .
\end{aligned}
$$

This timing ambiguity problem can be resolved in the $Y$ and $U$ coordinates in the same way. There still remains a spatial ambiguity if only $x$ and $y$ are used, since $X_{7}, X_{2}$, and $Y_{1}, Y_{2}$ can be coinbtned in four ways to give a point $X_{j}, Y_{j}$. This ambiguity is removed by using the redundant coordinate information.

Figure 11 shows another case. Here two events occur at different times, $t_{\mathrm{Cl}}$ and $t_{\mathrm{C} 2}$, but at a separation that is smaller than the delay line total propagation time. The reconstruction of the events from the data is done in a s!milar way to that discussed with $F i g .70$, except that an additional oatum, $t_{C 2}$, must be utilized. The analysis procedure is to search for triplets, $t_{A 1}, t_{B J}, t_{C k}$, such that

$$
\left(t_{A i}-t_{C k}\right)+\left(t_{B j}-t_{C k}\right)=T \text {. }
$$

The Identifled triplets can then be analyzed to determine $x_{Y}$, etc.

The 1 ines representing pulse trajectories in Figs. 9,10 , and 17 are del tberately made broad in order to represent the tinite "size" of pulses on the delay line. These pulses are typically such that, at the delay ifne output, a minimum separation of the order of $100 \mathrm{~ns}$ is necessary to allow individual detection of pulses that occur in pairs. If the vertex of the trajectories for one event lies within the broad trajectory of another event, it may not be possible to resolve them.

\section{References}

1. R. Grove, I. Ko, B. Leskovar, and V. Perez-Mendez, "Phase Compensated Electromagnetic Delay Lines for W1re Chamber Readout," Nucl. Instr. Methods 99, 381-385 (1972).

2. Muon Identification using Multiwire Proportional Chambers," National Accellerator Laboratory. Batavia, Illinots, Report TM358 (unpublished).

3. S. Parker and R. Jones, "External Muon Identifier Development: Haif Meter Proportional Chamber Test Results, "Lawrence Berkeley Laboratory Report LBL-797, May 1972 (unpublished).

4. Pages 247ff., of G. Charpak, "Evaluation of the Automat ic Spark Chamber," Ann. Rev. Nucl. Sci. 20, 195-254 (1970).

5. The BD-011 Branch Driver for the PDP-11 is manufactured by EG\&G/ORTEC, 100 Midland Road, Oak R\&dge, Tennessee. 
6. L. Q. Biswell and R. E. Rajala, "A MIcroprogramed Branch Driver (MBD) for a PDP-11 Computer,"Los Alamos Scientific Laboratory Report LA4916, Apri1 1972 (unpublished).

7. F. Kirsten, "Operational Description of Dfgitizers $22 \times 2140$ ror the EMI Muiti-wire Proportional Chambers with Delay-1 Ine Readout," Lawrence Berkeley Laboratory Engineering Note EET-1394, Apri1 1972 (unpubilished).

8. U.S. AEC Report TID-25875 (EUR-4100e), "CAMAC, A Modular Instrumentation System for Data Handling, Revised Description and Specification," July 1972. 
Fig. 1. A schematic representation of a MAPC and its three delay 1ines. The wires of each of the three planes are extended from the chamber so as to capacitively couple to the delay line. The labeling of the signal ports -- $A$, $B, E$, etc. - corresponds to Fig. 9.

Fig. 2. A schematic of the preamplifier design that uses an integrated circuit input stage. The signal-inverting IInks are shown connected for positive input signals; they are crossed over for negative signals. Because of the relatively high input current of the 733 , resistor $R 1$ must be chosen to be equal to the do source resistance $\left(Z_{0} / 2\right.$ for line terminated at both ends) plus 200 ohms.

Fig. 3. A block diagram of a discriminator. Seven identical channels are built in a NIM module.

Fig. 4. a) A simpliffed circuit of the zerocross detector used In the discriminator. A11 five transistors are contained in the CA30B6 integrated circuit. b) I-V characteristic and load line for the tunnel diode.

Fig. 5. A block diagram of the 7-channel digitizer module.

Fig. 6. Bit formats of words read from the digitizer:

a) The module identification word;

b) A data word.

Bit number 16, the most significant bit, differentiates between identification and data words.

Flg. 7. Block diagram of a complete MWPC readout system using the components described in the report.

FIg. 8. Sequence of major states in the process of digitizing data from a single beam spill.

Fig. 9. A diagram that illustrates the time relationships among pulses arising from an event in the MAPC.

Fig. 10. A time diagram (x-coordinate only) iliustrating two events occurring simultaneousiy in the .uPC at time $t_{\mathrm{Cl}}$.

Fig. 11. I1lustration of a situation in which two events occur at different times and at different locations. 


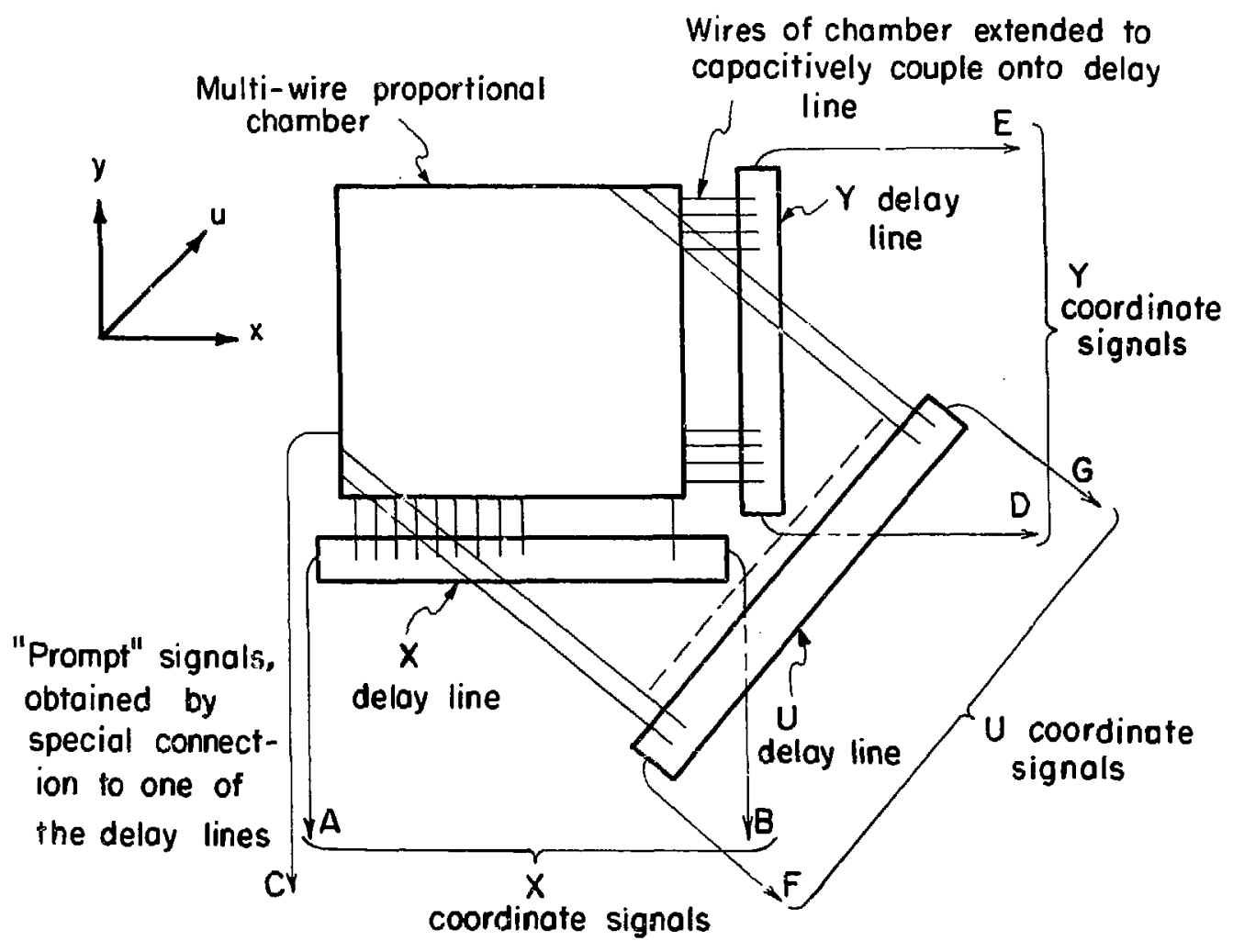




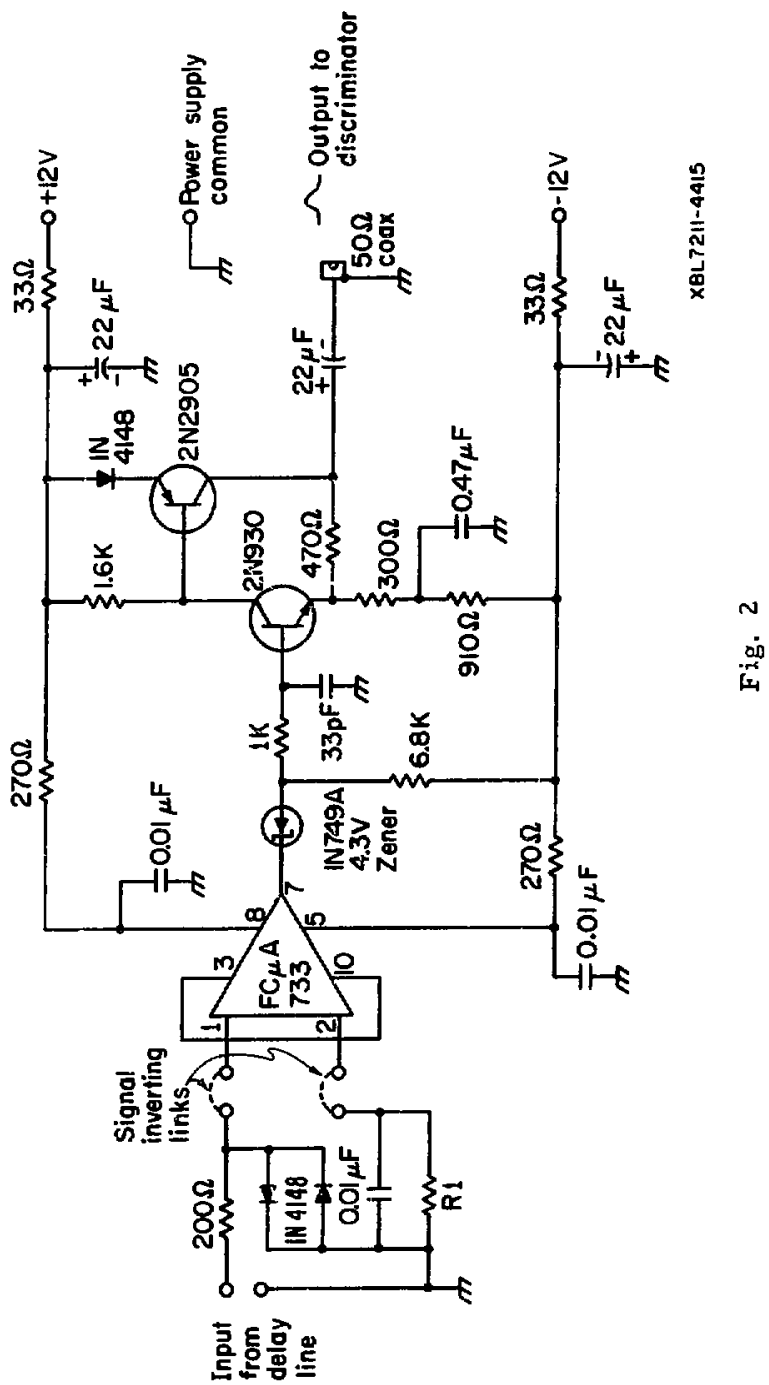



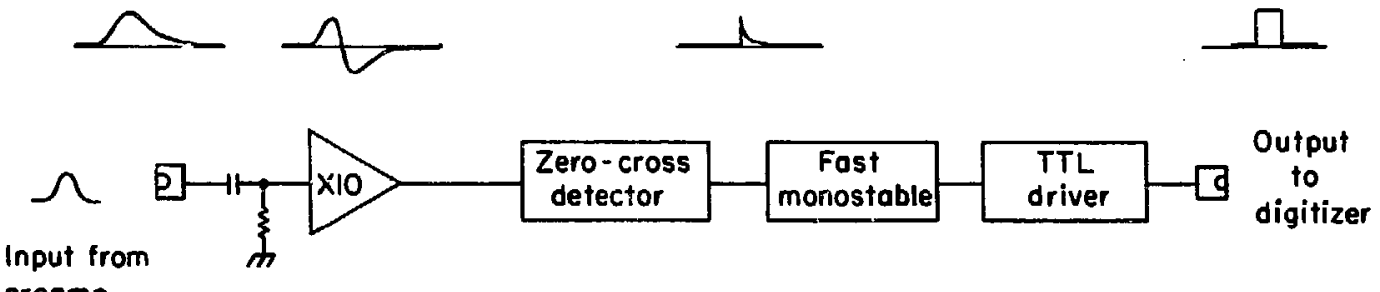

XBL $7211-44 ! 4$

Fig. 3 


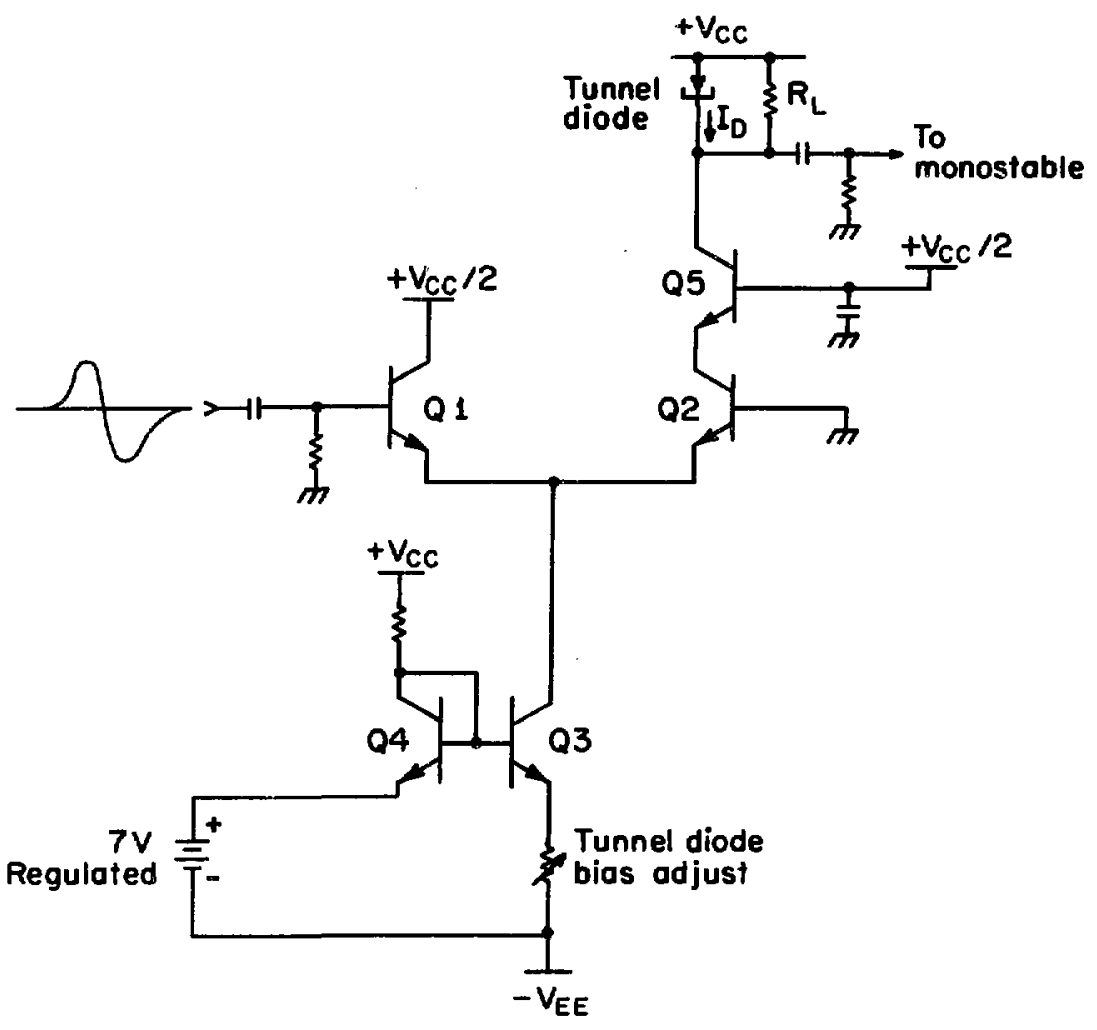

XBL72II-4413

Fig. $4 a$ 


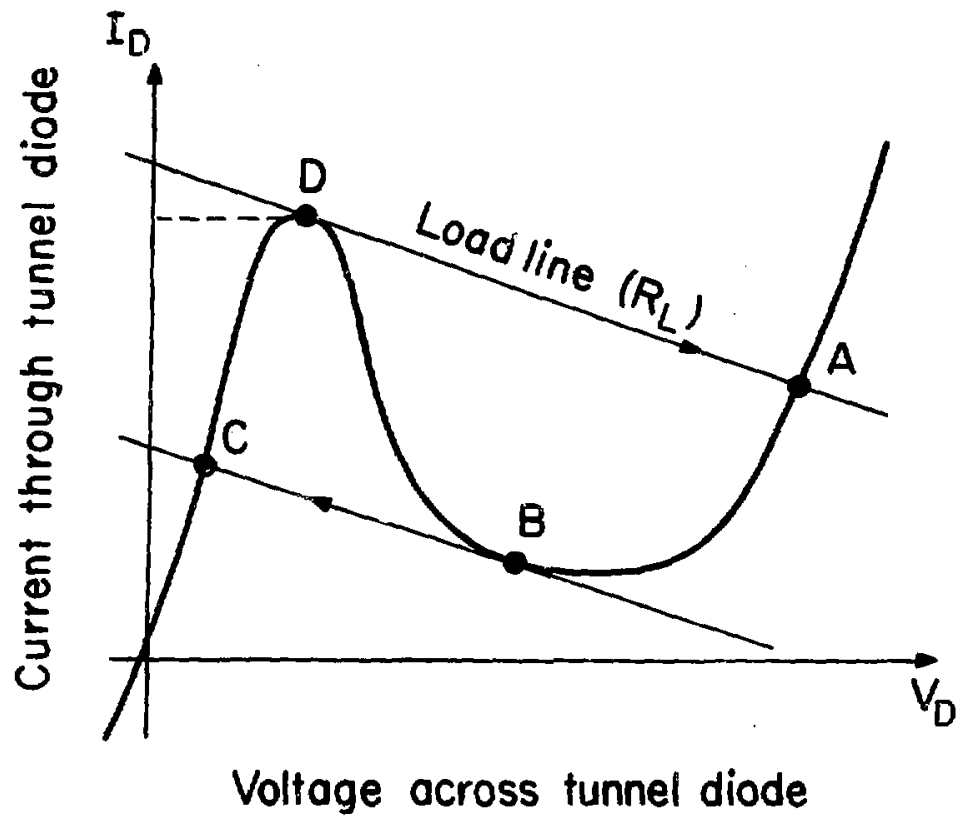

XBL 7211-4412

Fig. $4 b$ 


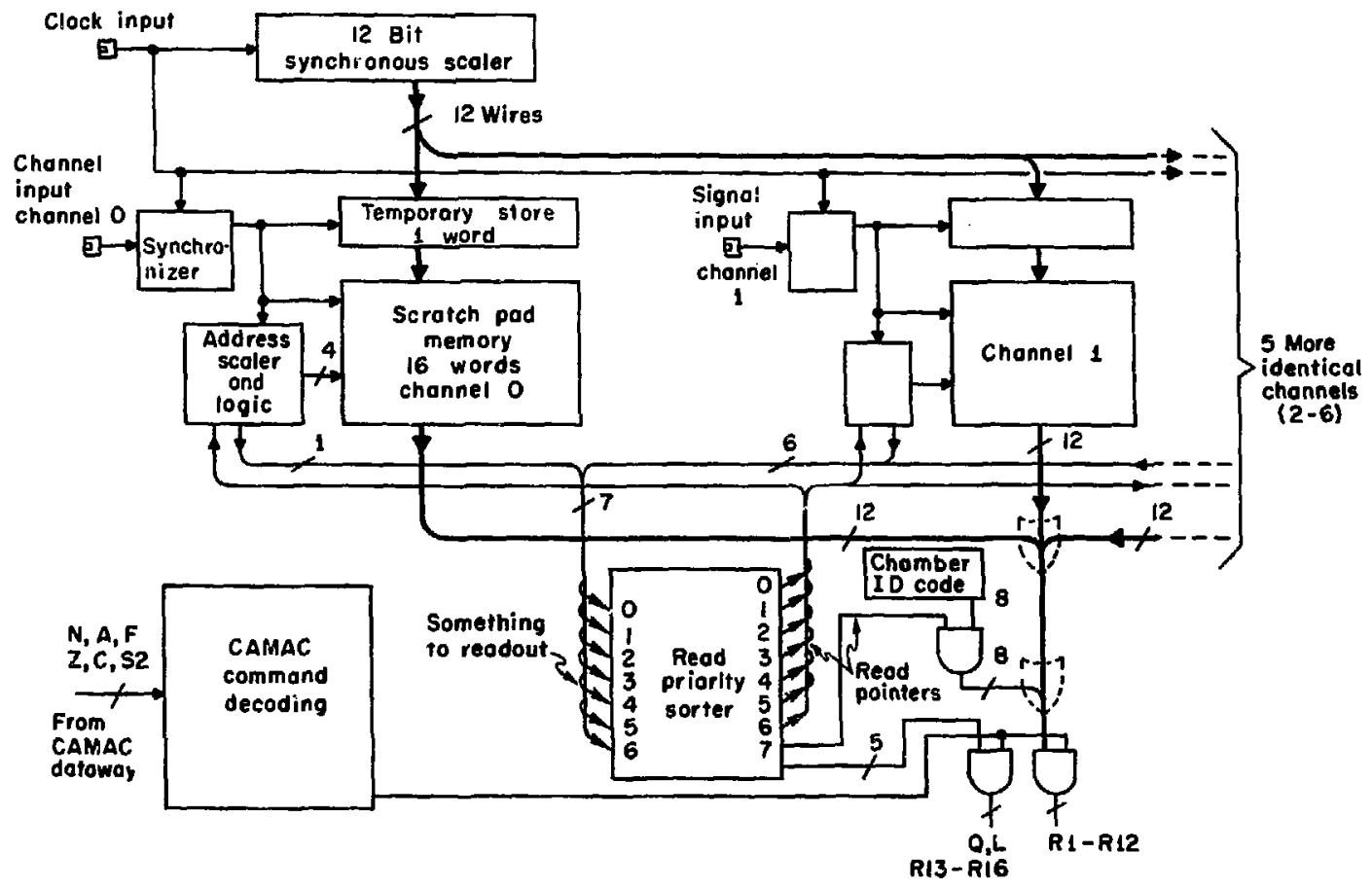

To CAMAC dotowoy

XBL72II-44II

Fig. 5 
(a) \begin{tabular}{l|l|l|}
16 & 15 & 98 \\
\hline 1 & Not significant & Module i.d. number \\
\hline
\end{tabular}

(b) \begin{tabular}{|l|l|l|l|}
16 & 15 & 13 & 12 \\
\hline 0 & $\begin{array}{l}\text { Channel } \\
\text { number }\end{array}$ & Dato \\
\hline
\end{tabular}

XBL7211-4410

Fig. 6 


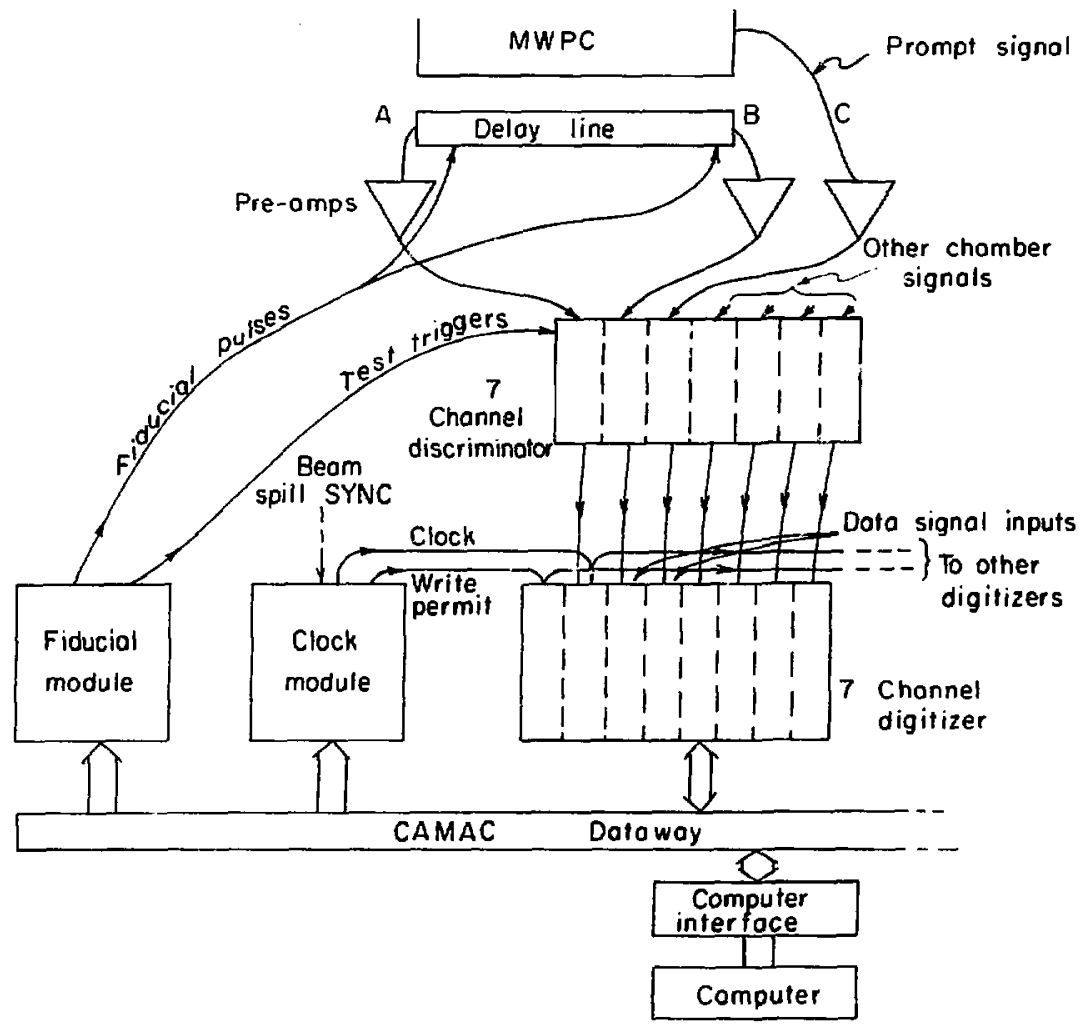

$X B L 7211-4409$

Fig. 7 


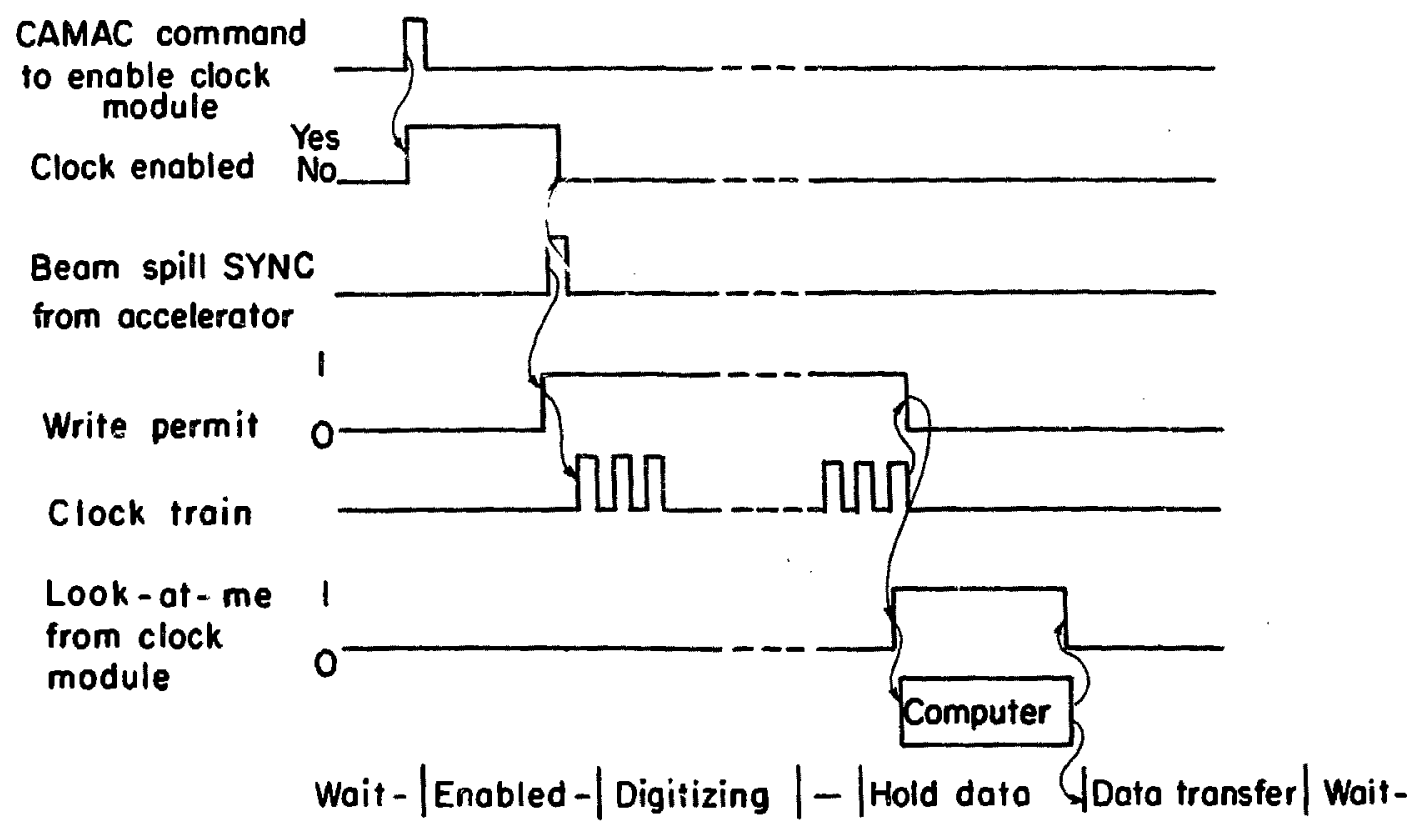




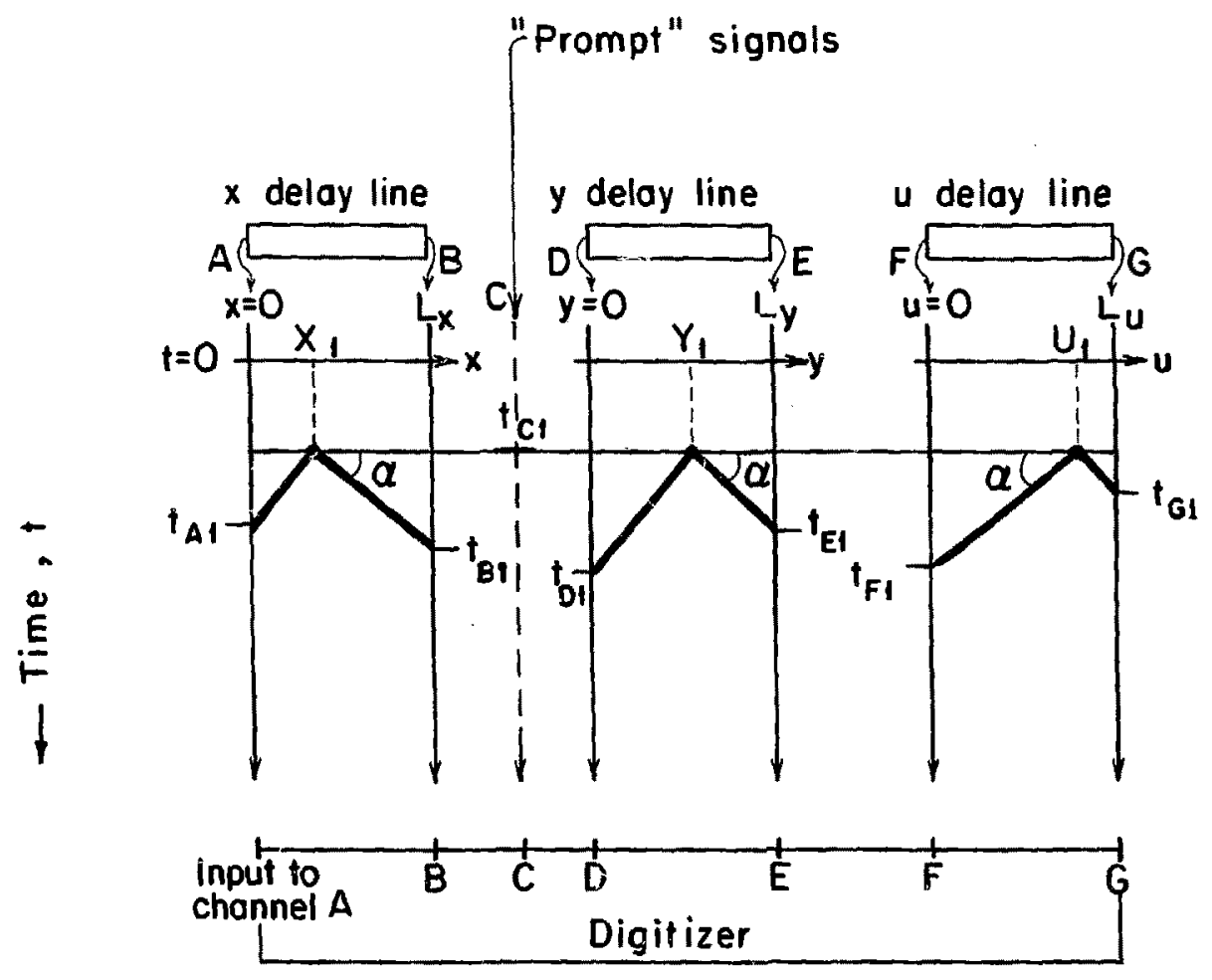


$-17-$

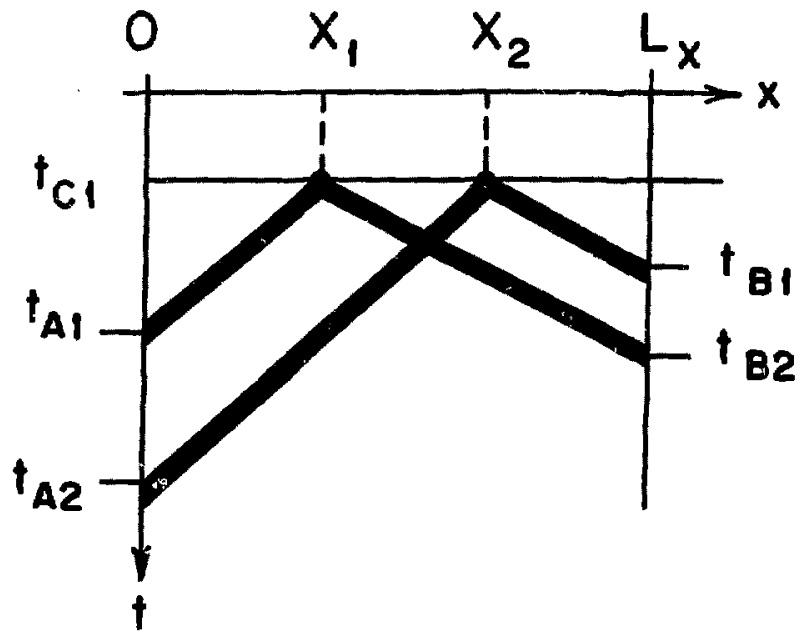

XBL72II-4406

Fig. 10 
$-18-$

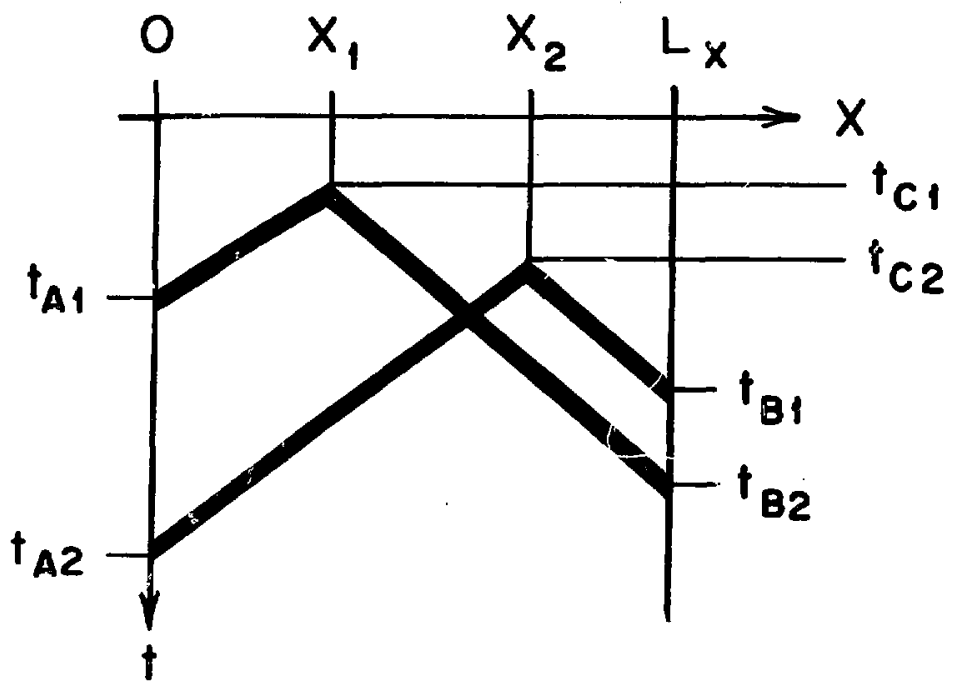

XBL72II-4407

Fig. 11 